\title{
Análisis de las mutaciones más frecuentes del gen BRCA1 (185delAG y 5382insC) en mujeres con cáncer de mama en Bucaramanga, Colombia
}

\author{
María Carolina Sanabria ${ }^{1,2}$, Gerardo Muñoz ${ }^{1,2}$, Clara Inés Vargas ${ }^{1,3}$ \\ 1 Departamento de Ciencias Básicas, Escuela de Medicina, Facultad de Salud, Universidad Industrial de \\ Santander, Bucaramanga, Colombia \\ 2 Grupo de Investigación Cáncer en Santander, Universidad de Santander, Bucaramanga, Colombia \\ 3 Grupo de Investigación Genética Humana, Universidad Industrial de Santander, Bucaramanga, Colombia
}

Introducción. El cáncer de mama es un problema de salud pública a nivel mundial y en Santander es la primera causa de morbimortalidad por cáncer en mujeres. Todo cáncer se considera una enfermedad genética y las mutaciones en los genes BRCA confieren un riesgo de $60 \%$ a $80 \%$ para el cáncer de mama. Este estudio consistió en buscar las dos mutaciones BRCA1 más frecuentes según la base de datos Breast Cancer Core Information.

Objetivo. Determinar la presencia de mutaciones específicas (185delAG, exón 2, y 5382insC, exón 20) en el gen BRCA1 en mujeres con cáncer de mama heredo-familiar, atendidas en los diferentes servicios de oncología de Bucaramanga, Colombia.

Materiales y métodos. La muestra incluyó 30 pacientes, de las cuales se obtuvo un consentimiento informado, un cuestionario dirigido y sangre venosa para los estudios moleculares. El análisis molecular se realizó mediante PCR-mismatch, para introducir o eliminar sitios de restricción, y digestión enzimática (Hinfl o Ddel).

Resultados. No se detectaron dos de las mutaciones más frecuentes en el gen $B R C A 1$ en las 30 pacientes estudiadas.

Conclusión. Se requieren más estudios en la región que abarquen la tamización de la totalidad del gen BRCA1, para hacer una mayor contribución al conocimiento de la epidemiología molecular del cáncer de mama en Bucaramanga, Santander, Colombia.

Palabras clave: neoplasias de la mama/genética, genes relacionados con las neoplasias, genes supresores, genes BRCA1, mutación de línea germinal, predisposición genética a la enfermedad.

Mutations in the BRCA1 gene (185delAG and 5382insC) are not present in any of the 30 breast cancer patients analyzed from eastern Colombia

Introduction. Breast cancer is considered a worldwide public health problem, and, in Santander Province, Colombia, it is the first leading cause of morbidity and mortality by cancer in women. All cancers are considered genetic diseases, and mutations in BRCA (BReast CAncer) genes raises the risk for breast cancer by $60 \%-80 \%$. The current study searched for the two most frequent $B R C A 1$ mutations reported in the Breast Cancer Core Information database.

Objective. The presence of specific mutations (185delAG, exon 2 and 5382insC, exon 20) was determined for the BRCA1 gene in women with familial/hereditary breast cancer.

Materials and methods. The sample included 30 female patients using the oncology services in Bucaramanga, eastern Colombia; an informed consent, a questionnaire and a blood sample were obtained from each. The molecular analysis was done with PCR-Mismatch, to detect the insertion or eliminatation of a restriction site, and enzymatic digestion methods (Hinfl or Ddel).

Results. Two of the most frequent BRCA1 mutations in the international database were not found in the 30 patients studied.

Conclusion. Additional mutation screening techniques are necessary involving the entire BRCA1 gene, are necessary in order to better characterize the molecular epidemiology of breast cancer in Bucaramanga, Santander, Colombia. 
Key words: Breast neoplasms/genetics; genes, neoplasm; genes, suppressor; genes, BRCA1; germ-line mutation, genetic predisposition to disease.

El cáncer de mama se considera un problema de salud pública en el mundo y constituye la primera causa de morbilidad por cáncer, 22\% (1), y la segunda causa de mortalidad, $15 \%$, en mujeres (2). En Santander, entre el 2000 y el 2004, la incidencia de cáncer de mama fue de 32,6 por 100.000 mujeres y la de cuello uterino fue de 19,8 por 100.000 mujeres (3).

El cáncer es una enfermedad multifactorial de base genética y para el cáncer de mama se ha identificado una serie de alteraciones genéticas en genes de baja y alta penetrancia. Los genes de alta penetrancia BRCA1 (4) y BRCA2 (5) son responsables de la mayoría de los cánceres de mama familiares/hereditarios; estos últimos constituyen del $5 \%$ al $10 \%$ del total de cánceres de mama (6) y en este grupo de pacientes la enfermedad se manifiesta en edades tempranas, de forma bilateral y asociada a cáncer de ovario (7).

La frecuencia de mutaciones $B R C A 1$ en cánceres de mama hereditarios depende del número de afectados y de la presencia adicional de cáncer de ovario en las familias; Ford et al. determinaron que $32 \%$ de las familias con cuatro afectados de cáncer de mama estaban ligadas al gen BRCA1 y que, cuando se adiciona al menos un caso de cáncer de ovario, este valor aumenta hasta $69 \%$ (8). Las mutaciones en los genes BRCA1 y $B R C A 2$, aumentan la probabilidad de sufrir cáncer de mama en $60 \%$ a $85 \%$ (8).

Se han identificado más de 600 mutaciones en cada uno de estos genes, lo que se debe a su gran tamaño; están conformados por 24 regiones (22 codificantes y 2 no codificantes), el gen BRCA1, y 27 regiones (26 codificantes

\footnotetext{
Correspondencia:

María Carolina Sanabria, Departamento de Ciencias Básicas, Escuela de Medicina, Facultad de Salud, Universidad Industrial de Santander, Carrera 32 No. 29-31, Bucaramanga, Santander, Colombia.

Teléfax: (577) 6455693

mariakmd24@yahoo.com

Recibido: 19/05/08; aceptado:16/10/08
}

y uno no codificante), el gen BRCA2. Según la base de datos de mutaciones $B R C A$ en cáncer de mama, Breast Cancer Information Core (BIC) del National Human Genome Research Institute/Nacional Institutes of Health (NHGRI/ $\mathrm{NIH}$, Bethesda), en total, las mutaciones más reportadas en este gen son la 185delAG, exón 2 , con una frecuencia de 16,4\% $(1,980 / 12,014)$ y la 5382insC, exón 20, con una frecuencia de $8,8 \%(1,063 / 12,014)$; las demás mutaciones que les siguen en frecuencia se presentan en menos de $2 \%$ cada una (9).

El origen de las mutaciones 185delAG y 5382insC sehacalculadoen 46y 38 generaciones, respectivamente, en judíos ashkenazi's, sefarditas y del Medio Oriente (Irán e Irak) desde antes de la primera dispersión judía, los cuales comparten un ancestro común. En la segunda dispersión judía, aquéllos establecidos en España (sefarditas) que migraron hacia el continente americano, podrían haber sido ya portadores de estas mutaciones; es similar el caso de los judíos que se asentaron en Alemania y más tarde en otros países de Europa Oriental (ashkenazi's) (10-12).

Lasinmigraciones deespañolesa Colombia desde el descubrimiento de América y de alemanes a partir del siglo XIX a Santander junto con Geo von Lengerke, han sido bien documentadas y podrían indicar la presencia de estas mutaciones en nuestra población. Estas dos mutaciones se han encontrado como fundadoras en población judía ashkenazi $(13,14)$ con unas frecuencias de $32,3 \%$ (185delAG) y 6,5\% (5382insC) en familias con cáncer de mama heredo-familiar (15). Además, la mutación 185delAG se ha hallado en Estados Unidos $(16,17)$, España $(11,18)$ y Chile $(19,20)$, mientras que la mutación 5382insC se ha encontrado en Alemania $(21)$, Italia $(22,23)$ y Polonia (24), entre otros países europeos (es la mutación más frecuente en este continente), y en población latina en Brasil (25).

En Colombia el único reporte publicado a la fecha consistió en el estudio de 53 familias colombianas con cáncer de mama u ovario hereditarios, en las cuales las mutaciones 3450delCAAG y A1708E 
del gen BRCA1 representaron el $100 \%$ de las mutaciones halladas en este gen, pero no se encontró ninguna de las dos mutaciones más frecuentes, según el BIC (26).

Mediante estudios sobre variabilidad genética en poblaciones de los departamentos de Santander y de Norte de Santander, usando marcadores haplotípicos, se determinó a partir del ADN mitocondrial (mtADN) que la contribución femenina está dada principalmente por mujeres nativas de Santander (92,9\%); mientras que, a partir del cromosoma $\mathrm{Y}$, se determinó que el aporte masculino está representado en su mayoría por linajes europeos $(86,6 \%)$. En esa contribución europea se encontraron marcadores genéticos judíos en $12,7 \%$, de los cuales, $7 \%$ correspondió al haplotipo Cohen, perteneciente al apellido judío más antiguo, Cohanim, que se encuentra tanto en judíos ashkenazi como en judíos sefarditas (comunicación personal, María Mercedes Torres).

Los orígenes de estas mutaciones y su gran frecuencia, la historia migratoria de nuestra región y los estudios de linajes mediante la tipificación haplotípica, motivaron el desarrollo de esta investigación.

En este estudio se buscaron las dos mutaciones reportadas con más frecuencia en el gen $B R C A 1$ según la base de datos $\mathrm{BIC}$, en un grupo de mujeres con cáncer de mama de Bucaramanga, Santander, con antecedentes familiares de cáncer de mama, ovario u otro tipo de cáncer relacionado con mutaciones en el gen BRCA1.

\section{Materiales y métodos}

\section{Pacientes}

Se llevó a cabo un estudio descriptivo de 30 casos de cáncer de mama en mujeres provenientes del departamento de Santander, Colombia, atendidas en cinco centros oncológicos.

La recolección se hizo entre marzo del 2006 y marzo del 2007, con los siguientes criterios de inclusión: i) mujeres de cualquier edad con diagnóstico clínico e histopatológico de cáncer de mama; ii) tener, al menos, un antecedente familiar en primero o segundo grado de cáncer de mama, ovario u otro tipo de cáncer relacionado con este gen como: cáncer de estómago, próstata, colon, útero o vía biliar (hígado, vesícula biliar, páncreas) (27-30), y iii) pacientes con cáncer de mama diagnosticado a los 36 años o antes, aun sin antecedentes familiares de cáncer en general. El criterio de exclusión fue el parentesco entre las participantes.

El proyecto fue aprobado por el Comité de Ética de la Universidad Industrial de Santander-Hospital Universitario de Santander y todas las pacientes firmaron un consentimiento informado.

Se diseñó un cuestionario dirigido para recopilar datos personales relacionados con factores sociodemográficos, antecedentes clínicos y antecedentes familiares.

\section{Análisis genético}

El análisis consistió en determinar la presencia o ausencia de las dos mutaciones más frecuentes en el gen BRCA1. El ADN se extrajo por salting out a partir de $4 \mathrm{ml}$ de sangre periférica anticoagulada con EDTA (31).

PCR-mismatch: esta técnica molecular se ha usado previamente para la búsqueda de las mutaciones específicas de interés en este estudio $(15,21)$, que consiste en usar un cebador con un cambio de base en su secuencia (cuadro 1) para incluirlo en los amplificados, creando o eliminando un sitio de restricción para una enzima determinada. La amplificación de ambos exones se hizo por separado en una mezcla de reacción con volumen final de $50 \mu \mathrm{l}$, que contenía una concentración final de ADN $50 \mathrm{ng} /$ $\mu \mathrm{l}, \mathrm{MgCl}_{2}(1,5 \mathrm{mM})$, dNTP (0,2 mM cada uno), cebadores $(0,4 \mu \mathrm{M}$ cada uno) y $0,2 \mathrm{U}$ de Tucan Taq polimerasa (Corporación Corpogen, Bogotá, Colombia). Los ciclos de amplificación fueron para el exón 2: desnaturalización inicial a $94{ }^{\circ} \mathrm{C}$ durante 3 minutos, 30 ciclos (desnaturalización a $94{ }^{\circ} \mathrm{C}$ durante 30 segundos, hibridación a $55^{\circ} \mathrm{C}$ durante 30 segundos y extensión a $72{ }^{\circ} \mathrm{C}$ durante 30 segundos), seguido de una extensión final a $72{ }^{\circ} \mathrm{C}$ durante 10 minutos y temperatura final de $4{ }^{\circ} \mathrm{C}$. Las condiciones para el exón 20 fueron similares a las usadas para el exón 2 , pero con una temperatura de hibridación de $61^{\circ} \mathrm{C}$ durante 30 segundos. 
Cuadro 1. PCR-mismatch y digestión enzimática para detección de las mutaciones 185delAG y 5382insC del gen BRCA1.

\begin{tabular}{|c|c|c|c|c|c|}
\hline Mutación & $\begin{array}{l}\text { Cebadores normal y } \\
\text { mismatch }(27,40)\end{array}$ & $\begin{array}{l}\text { T.H. } \\
{ }^{\circ} \mathrm{C}\end{array}$ & Amplicón & $\begin{array}{l}\text { Enzima } \\
\text { restricción }\end{array}$ & $\begin{array}{l}\text { Fragmentos } \\
\text { digeridos } \\
\text { (pb) }\end{array}$ \\
\hline $\begin{array}{l}\text { 185delAG } \\
\text { Exón } 2(15)\end{array}$ & $\begin{array}{l}\text { F 5'-GAA GTT GTC ATT TTA TAA ACC TTT-3' } \\
\text { R 5'-TGA CTT ACC AGA TGG GAG AC-3' }\end{array}$ & 55 & $\begin{array}{l}\mathrm{N}: 170 \mathrm{pb} \\
\mathrm{M}: 168 \mathrm{pb}\end{array}$ & $\begin{array}{l}\text { Hinf I } \\
\text { 5'G^ANTC } \\
\text { 3'CTNA^G }\end{array}$ & $\begin{array}{l}N: 150+20 \\
\quad M: 168\end{array}$ \\
\hline $\begin{array}{l}\text { 5382insC } \\
\text { Exón } 20(21)\end{array}$ & $\begin{array}{l}\text { F 5'CCA AAG CGA GCA AGA GAA TCT C-3' } \\
\text { R 5'GGG AAT CCA AAT TAC ACA GC-3' }\end{array}$ & 61 & $\begin{array}{l}\mathrm{N}: 270 \mathrm{pb} \\
\mathrm{M}: 271 \mathrm{pb}\end{array}$ & $\begin{array}{c}\text { Ddel } \\
\text { 5'C^TNAG } \\
\text { 3'GANT^C }\end{array}$ & $\begin{array}{l}\mathrm{N}: 214+36+20 \\
\mathrm{M}: 235+36\end{array}$ \\
\hline
\end{tabular}

Cebadores normal y mismatch: las bases en rojo corresponden a las bases mismatch

T.H. ${ }^{\circ} \mathrm{C}$ : temperatura de hibridación de los cebadores para la PCR-mismatch correspondiente

Amplicón: tamaños en pares de bases $(\mathrm{pb})$ de los amplificados normales (N) y mutados (M)

Digestión enzimática: esta técnica complementa la anterior (PCR-mismatch) y consiste en que los amplificados obtenidos con el mismatch se someten a un proceso de digestión con determinadas enzimas para el reconocimiento del sitio de restricción, lo que permite diferenciar un alelo normal de uno mutado. Para la mutación 185delAG, se usó la enzima $\mathrm{Hinfl}\left(37^{\circ} \mathrm{C}\right.$ durante 20 horas) y, para la mutación 5382insC, la enzima Ddel ( $37^{\circ} \mathrm{C}$ durante 16 horas).

Los fragmentos se analizaron en gel de agarosa de bajo punto de fusión (LMP, low melting point) al $4 \%$. Para el alelo normal del exón 2 , se observó un fragmento digerido de $150 \mathrm{pb}$ y, para el alelo mutado, un fragmento de $168 \mathrm{pb}$ (15) (cuadro 1). Para el alelo normal del exón 20 se observó un fragmento digerido de $214 \mathrm{pb}$ y, para el alelo mutado, un fragmento de $235 \mathrm{pb}$ (21) (cuadro 1). La actividad de las enzimas se probó con la digestión del plásmido PUC18 y del ADN directo. Como controles positivos se usaron muestras con las respectivas mutaciones, donadas por el Laboratorio NE Thames, UK. Como control negativo se usó el ADN extraído a partir de la línea celular MCF-7 (ATCC № HTB-22 ${ }^{\mathrm{TM}}$ ) (32). Para confirmar los hallazgos negativos del exón 2 , cuatro muestras elegidas al azar se analizaron mediante secuenciación directa en el Laboratorio de la Universidad de Porto, IPATIMUP, Portugal. La secuenciación de la totalidad del gen, incluyendo el exón 20 en esta cohorte, está pendiente para futuros trabajos.

\section{Resultados}

Se incluyeron 30 pacientes, con un promedio de edad al momento del diagnóstico de 49,6 años, de las cuales, $16,7 \%(5 / 30)$ eran menores de 37 años (27 a 36 años). El 43,3\% (13/30) de las pacientes pertenecía a estrato socioeconómico bajo. Tres pacientes refirieron una edad de la menarquia a los 11 años o menos. La tercera parte consumió hormonas exógenas, como anticonceptivos orales o terapia de reemplazo hormonal, por un periodo comprendido entre ocho meses y 18 años (6,52 años en promedio). Un porcentaje significativo de mujeres tuvieron su primer parto antes de los 20 años (43,3\%). El número de embarazos a término más frecuente entre las pacientes fue de tres y cuatro (14/30; $46,6 \%$ ) y dos pacientes de 27 y 59 años refirieron ser nulíparas.

Con respecto a la historia familiar, cuatro de las cinco pacientes menores de 37 años no reportaron familiares afectados por ningún tipo de cáncer. El $86,7 \%(26 / 30)$ de las pacientes tenían, al menos, un antecedente de cáncer de mama, ovario u otros cánceres relacionados con mutaciones en el gen BRCA1; de éstas, $20 \%$ (6/30) tenía sólo antecedentes familiares de cáncer de mama, $26,7 \%(8 / 30)$ tenían familiares afectadas por cáncer de mama y por otros tipos de cáncer relacionados y $40 \%(12 / 30)$ de las pacientes refirieron sólo familiares afectados por otros tipos de cáncer diferente al de mama, relacionados con mutaciones en el gen BRCA1, como: cáncer 
Cuadro 2. Descripción de las pacientes según sus antecedentes familiares.

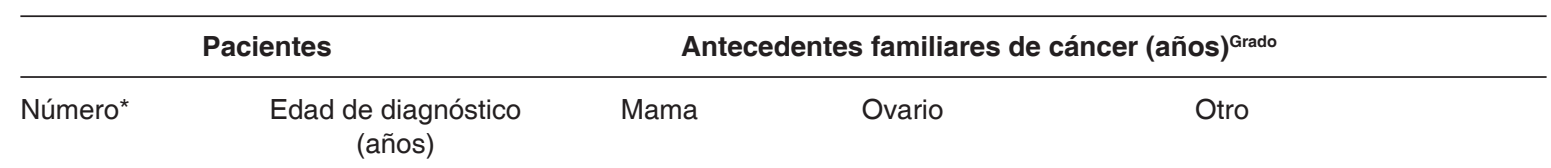

Cáncer de mama <37 años sin antecedentes familiares (4/30)

$\begin{array}{lcc}1 & 35 & \\ 2 & 27 & \\ 3 & 32 & \\ 4 & 36 & \\ \text { Sólo antecedentes familiares de cáncer de mama }(6 / 30) \\ 5 & 46 & (35)^{2} \\ 6 & 35 & (26,62)^{1} \\ 7 & 39 & (72)^{2} \\ 8 & 53 & (44)^{1} \\ 9 & 38 & (50)^{2} \\ 10 & 50 & (48)^{1}\end{array}$

Antecedentes familiares de cáncer de mama y otros tipos (8/30)

\begin{tabular}{|c|c|c|c|c|}
\hline 11 & 40 & $(40) 1$ & & Próstata $(70)^{1}$ \\
\hline 12 & 46 & $(46) 1$ & & $\begin{array}{l}\text { Tiroides }(40)^{1} ; \text { gástric(60)2; no } \\
\text { especificado }(60)^{2}\end{array}$ \\
\hline 13 & 67 & $\operatorname{LM}(35,55)^{2}$ & & $\begin{array}{l}\text { LM: colon }(59)^{1} \text {; esófago }(60)^{2} \text {; leucemia } \\
(50,60)^{2} \text {; gástrico }(50,60)^{2} \\
\text { LP: esófago-gástrico }(83)^{1}\end{array}$ \\
\hline 14 & 45 & $\left(48^{\star \star}, 52,64\right)^{1}$ & & Útero $\left(48^{* \star}\right)^{1}$ \\
\hline 15 & 55 & $(?, ?)^{2}$ & & $\begin{array}{l}\text { Gástrico }(60)^{2} \text {; hepático }(70,70)^{2} \text {; } \\
\text { no especificado }(50)^{2}\end{array}$ \\
\hline 16 & 60 & $\operatorname{LM}(50,56,60)^{1}$ & & LP: tejidos blandos $(34)^{2}$ \\
\hline \multirow[t]{2}{*}{17} & 52 & $\operatorname{LM}(30)^{2}$ & & LM: gástrico $(40)^{2}$; tiroides $(37)^{2}$ \\
\hline & & $\operatorname{LP}(52,60)^{2}$ & LP (38)2 & $\begin{array}{l}\text { LP: gástrico }(80)^{2} \text {; páncreas }(86)^{2} \text {; } \\
\text { piel }(84)^{2}\end{array}$ \\
\hline \multirow[t]{2}{*}{18} & 44 & $(45)^{1}$ & & LP: hepático $(70)^{2}$ \\
\hline & & $\operatorname{LP}(46,50)^{2}$ & & LM: pulmonar (46)2; hepático $(53)^{2}$ \\
\hline
\end{tabular}

Sólo antecedentes familiares de otros tipos de cáncer diferente al de mama (12/30)

19

67

$\begin{array}{ll}20 & 70 \\ 21 & 59 \\ 22 & 46 \\ 23 & 41 \\ 24 & 47 \\ 25 & 45 \\ 26 & 61 \\ 27 & 68 \\ 28 & 67 \\ 29 & 41 \\ 30 & 75\end{array}$

Cuello uterino $(48,55) 1$; nasal $(80,48)^{1-2}$; próstata $(18,65)^{1-2}$; gástrico $(33)^{2}$;

no especificado $(40)^{2}$

Próstata $(60,70)^{1}$

Vesícula biliar $(72)^{1}$

Hepático $(68)^{1}$

Gástrico (?) ${ }^{2}$

Colon $(68)^{2}$

Útero $(83)^{2}$

Próstata (?) ${ }^{1}$; no especificado $(<40)^{2}$

Esófago (70) ${ }^{1}$; colon (38) ${ }^{2}$

Cerebral $(56)^{1}$; útero $(22)^{2}$; gástrico $(50)^{2}$ Útero $(50)^{2}$

Colon (50)

Número*: el número del paciente en esta lista no corresponde con el sistema de codificación de pacientes-muestras.

Antecedentes familiares de cáncer (años) Grado: el número que está entre paréntesis es la edad de diagnóstico de los familiares afectados; el (?) indica que no conocía la edad de los familiares al momento del diagnóstico; el superíndice corresponde al grado de parentesco con el probando, primer ${ }^{1} \mathrm{o}$ segundo ${ }^{2}$ grado; LM (línea materna); LP (línea paterna); ${ }^{*}$ corresponde a una misma paciente con cáncer de mama y cáncer de útero. 
de estómago, próstata, colon, útero o vía biliar (hígado, vesícula biliar, páncreas), algunos de los cuales incluyeron, además, otros cánceres como leucemias, cáncer de nariz, cuello uterino, sistema nervioso central, tiroides, pulmón, tejidos blandos-piel, y otros no especificados. Sólo una paciente (número 17), de 52 años al momento del diagnóstico, presentó un familiar afectado con cáncer de ovario (cuadro 2).

Con respecto a las características histopatológicas, el tipo más reportado fue el carcinoma ductal infiltrante moderado/poco diferenciado y sólo una paciente fue diagnosticada en estadio in situ de tipo ductal. El diagnóstico definitivo más frecuente fue el de carcinoma ductal, $76,7 \%$ (23/30), que incluyó: i) carcinoma ductal infiltrante muy diferenciado (tres), ii) carcinoma ductal infiltrante moderado/poco diferenciado (19) y iii) carcinoma ductal in situ (uno). El grado histológico Bloom-Richardson más predominante fue el moderado y el $46,7 \%$ de los tumores eran receptores hormonales negativos, mientras que el $30 \%$ correspondieron a receptores hormonales positivos (cuadro 3).
En cuanto a las características clínicas, el reporte mamográfico BIRADS IV, indicador de sospecha para malignidad de la mama, fue el más frecuente $(66,7 \%)$ y en $43,3 \%$ de las pacientes el estadio al momento del diagnóstico fue $\mathrm{E}$ IIIB.

Deacuerdo con los resultados correspondientesal análisis molecular del exón 2, no se evidenciaron mutaciones; en todos los casos se observó una banda a la altura de $150 \mathrm{pb}$ correspondiente al alelo normal (figuras 1A y 1B). La negatividad de la muestra 004 (flecha roja) se corroboró mediante secuenciación directa (figura 2). En el análisis correspondiente al exón 20 , tampoco se encontró mutación y en todos los casos se observó una banda de 214 pb correspondiente al alelo normal (figuras $3 \mathrm{~A}$ y $3 \mathrm{~B}$ ).

\section{Discusión}

Debido al alto riesgo para cáncer de mama y ovario asociado a mutaciones en los genes BRCA1-2, su análisis molecular se ha popularizado, especialmente en los grupos de alto riesgo para la enfermedad que son, principalmente, aquéllos con múltiples antecedentes familiares de cáncer

Cuadro 3. Descripción de las características histopatológicas del tumor de mama.

\begin{tabular}{lcc}
\hline Características histopatológicas & Número de pacientes & $\begin{array}{c}\text { Frecuencia } \\
\text { (\%) }\end{array}$ \\
\hline Biopsia & $\mathbf{3 0}$ & 63,3 \\
Carcinoma ductal infiltrante mod/poco diferenciado & 19 & 10 \\
Carcinoma ductal infiltrante muy diferenciado & 3 & 3,3 \\
Carcinoma ductal in situ & 1 & 13,3 \\
Carcinoma lobulillar infiltrante mod/poco diferenciado & 4 & 6,7 \\
Carcinoma lobulillar infiltrante muy diferenciado & 2 & 3,3 \\
Carcinoma túbulo-lobulillar & 1 & 16,7 \\
Grado histológico Bloom-Richardson & & 46,7 \\
Bajo grado & 5 & 16,7 \\
Moderado grado & 14 & 20,0 \\
Alto grado & 5 & 46,7 \\
No dato & 6 & 6,7 \\
Receptores hormonales & & 0,0 \\
ER (-) y PR (-) & 14 & 30,0 \\
ER (-) y PR (+) & 2 & 16,7 \\
ER (+) y PR (-) & 0 & 9 \\
ER (+) y PR (+) & 9 & 5 \\
Sin dato & 5 & \\
\hline
\end{tabular}

Receptores hormonales: receptores de estrógenos (ER)y receptores de progestágenos (PR)

Positivos (+)

Negativos (-) 


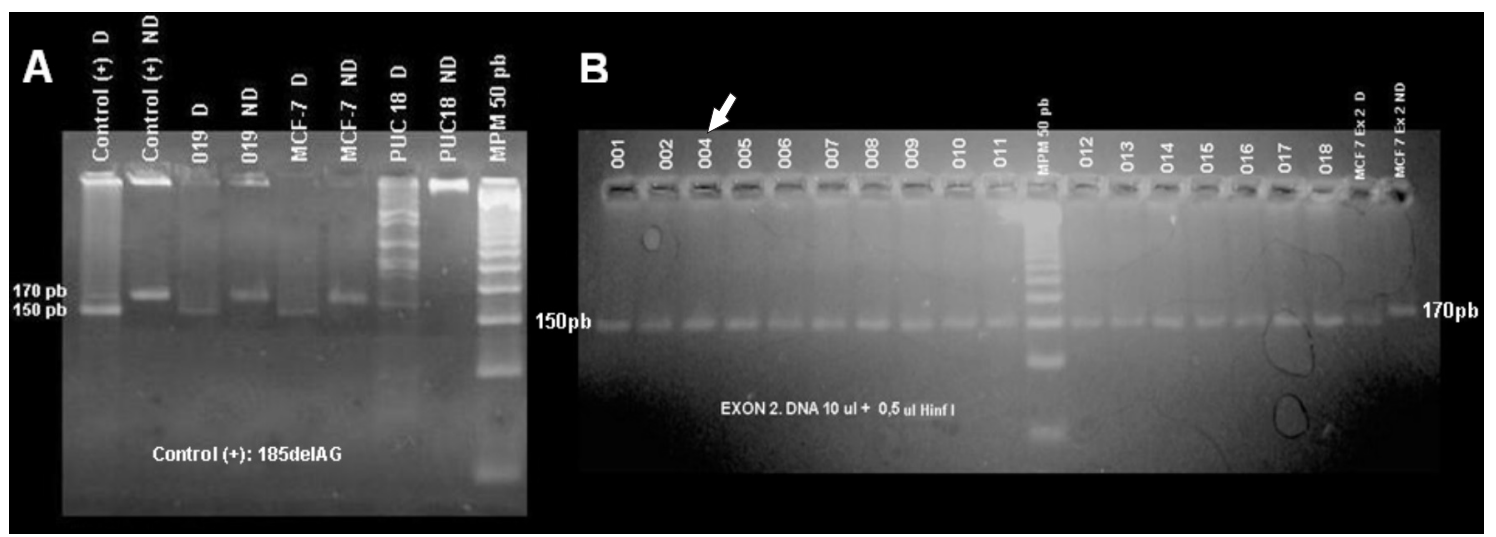

Figura 1. Análisis de restricción enzimática del exón 2 del gen $B R C A 1$.

En la figura $1 \mathrm{~A}$ se observan los amplificados correspondientes al exón 2, con la enzima -digeridos (D)- y sin la enzima -no digeridos (ND)-, en un gel de agarosa LMP al 4\%. La muestra $019 \mathrm{D}$ representa el genotipo homocigoto negativo para la mutación 185delAG, con una banda a la altura de $150 \mathrm{pb}$, que se corresponde con el control negativo (MCF-7). Por otra parte, el control (+) D se muestra heterocigoto para la mutación 185delAG, con dos bandas de 170 pb y 150 pb para los alelos mutado y normal, respectivamente.

Control (+) D: control positivo digerido; Control (+) ND: control positivo no digerido; MCF-7 D: control negativo digerido; MCF-7 ND: control negativo no digerido.

pUC18: plásmido usado para probar la actividad de la enzima Hinfl.

MPM $50 \mathrm{pb}$ : marcador de peso molecular de $50 \mathrm{pb}$.

En la figura 1B se observan algunas muestras analizadas para la mutación 185delAG. La negatividad para la mutación de la muestra 004 (flecha blanca) fue corroborada mediante secuenciación directa (figura 2).

de mama u ovario, pero también en los casos de cáncer de mama de aparición temprana, bilateralidad del tumor primario, y cáncer de mama y ovario en la misma persona (33).

En esta cohorte de pacientes con cáncer de mama, $86,7 \%$ tenían, al menos, un familiar en primero o segundo grado afectado por cáncer de mama, ovario $u$ otro tipo de cáncer relacionado con BRCA1 (27-30) y 13,3\% correspondía a pacientes con cáncer de mama diagnosticado antes de los 37 años, sin historia familiar; todos los anteriores se tomaron como indicadores de una posible susceptibilidad heredo-familiar (cuadro 2).

En otros estudios se han usado criterios similares, por ejemplo, Ikeda et al. (34) incluyeron pacientes con cáncer de mama con, al menos, un familiar en primer grado con cáncer de mama o cáncer de ovario, para el análisis de genes $B R C A$ en población japonesa, y encontraron mutaciones BRCA1 en $13,3 \%$ (15/113) de las pacientes; en otros, como el realizado en el Reino Unido por Peto et al. (35), se analizaron 254 pacientes con cáncer de mama de aparición temprana, antes de los 36 años, y encontraron mutaciones BRCA1 en $3,5 \%(9 / 254)$, de los cuales, la mutación 5382insC correspondió a 0,4\% (1/254). A su vez, en el estudio realizado por Ruiz-Flórez et al. (36) se incluyó a 32 mujeres mexicanas con cáncer de mama diagnosticado a los 35 años o antes y se encontraron mutaciones $B R C A 1$ en $3,13 \%$ $(1 / 32)$.

En términos de resultados del análisis molecular, en nuestra cohorte de pacientes no se encontró la mutación de interés (185delAG) en el exón 2. En otro estudio realizado por Trincado et al. en Chile (37), en el que incluyeron 15 pacientes con cáncer de mama familiar y 40 de tipo esporádico, tampoco se encontró esta mutación. Sin embargo, en estudios más recientes en esta misma población, se logró identificar la mutación en bajas frecuencias. Jara et al. (20) analizaron esta mutación en 382 mujeres sanas con, al menos, dos familiares afectados por cáncer de mama, y encontraron una frecuencia de $0,26 \%(1 / 382) y$, posteriormente, Jara et al. (19) la encontraron en $3,12 \%(2 / 63)$ de familias 
seleccionadas por criterios de alto riesgo para la enfermedad.

La mutación 5382insC del exón 20, BRCA1, tampoco se encontró en las 30 pacientes incluidas en este estudio. En Latinoamérica se han llevado a cabo algunos estudios en su búsqueda, como lo hicieron Jara et al. (38) quienes no encontraron la mutación en 382 mujeres sanas con, al menos, dos familiares afectados por cáncer de mama. Por su parte, Dufloth et al. (25), en una muestra de 31 pacientes de Brasil, con cáncer de mama e historia familiar, encontraron la mutación en $3,2 \%(1 / 31)$.

En Colombia, el estudio realizado por Torres et al. evidenció dos mutaciones fundadoras en el gen $B R C A 1$ en 8/53 familias de alto riesgo para cáncer de mama (según el análisis de haplotipos), previamente reportadas en el $\mathrm{BIC}$ en poblaciones europeas y latinoamericanas; sin embargo, ninguna de éstas corresponden a las más frecuentes en esta base de datos. De otra parte, los hallazgos del presente trabajo muestran que las mutaciones más frecuentemente reportadas en el BIC no se encontraron, lo que apoya la necesidad de realizar tamizaciones genéticas que abarquen la totalidad del gen BRCA1.

Con respectoalascaracterísticas histopatológicas y clínicas de esta cohorte, se encontró que el tipo histopatológico más frecuente fue el carcinoma ductal $(76,7 \%)$, acorde con el $77,6 \%$ reportado en la literatura (39). El $60 \%$ de las pacientes analizadas fueron diagnosticadas en E IIIA (16,7\%) y IIIB (43,3\%), lo cual indica que, lastimosamente, en nuestro medio se sigue detectando el cáncer de mama en etapas muy avanzadas, cuando las posibilidades de supervivencia y curación están reducidas; lo anterior corresponde con el $71,5 \%$ reportado en estudios locales (39).

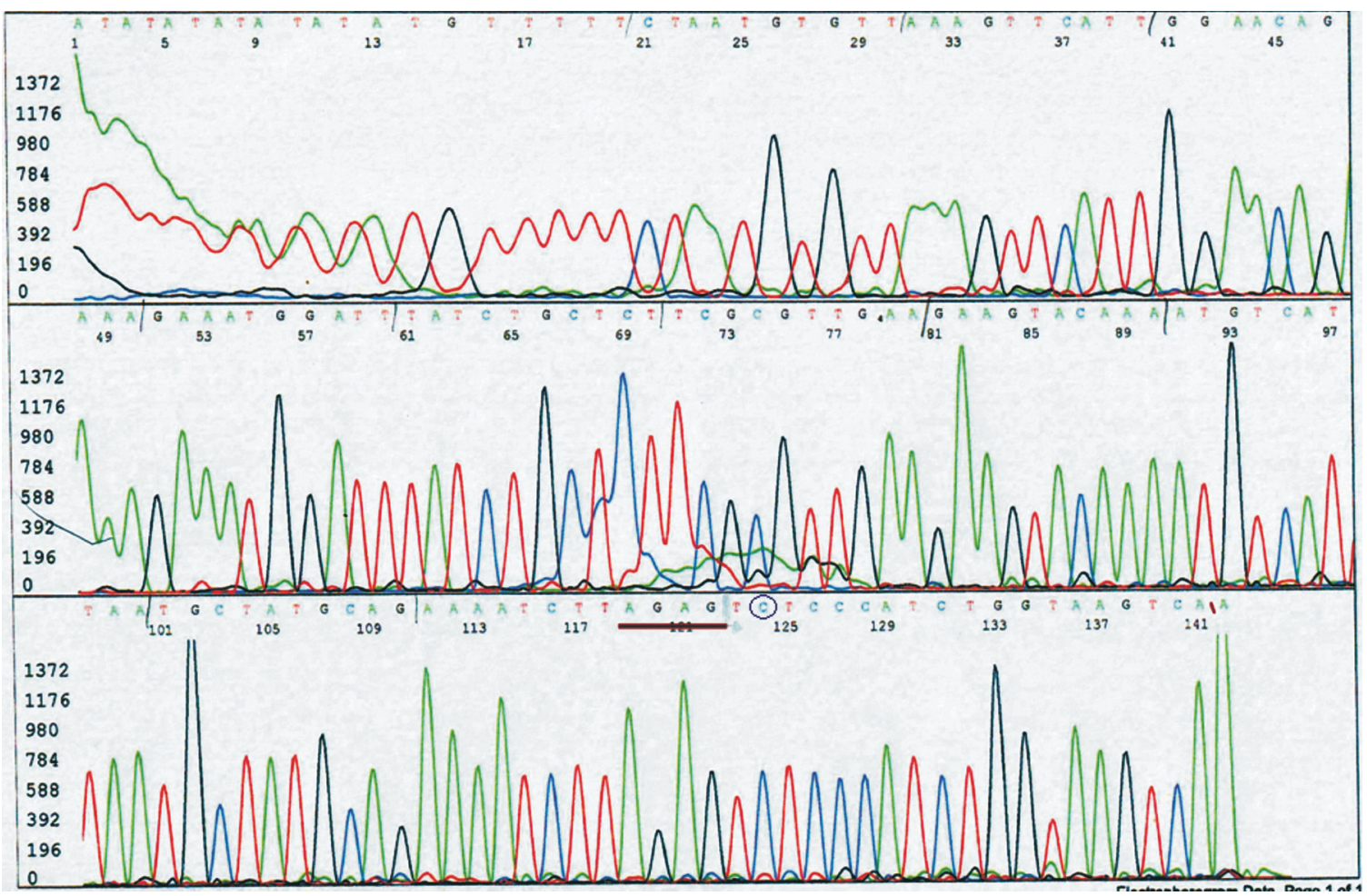

Figura 2. Secuenciación directa del exón 2 del gen BRCA1, muestra 004

Secuenciación directa del exón 2 de la muestra 004, la línea roja subraya la secuencia normal AG AG y el círculo morado señala el cambio de base ( $G$ por $C$ ) generado mediante la técnica de PCR-mismatch. 


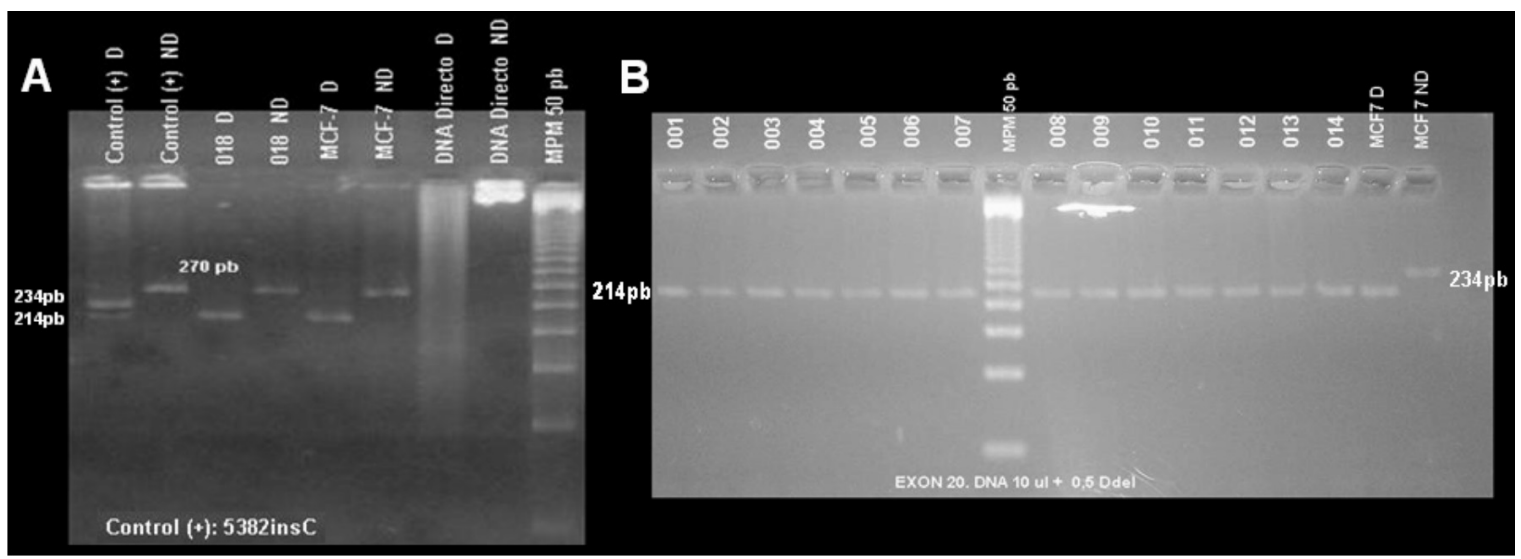

Figura 3. Análisis de restricción enzimática del exón 20 del gen BRCA1

En la figura 3A se observan los amplificados correspondientes al exón 20 con la enzima -digeridos (D)- y sin la enzima -no digeridos (ND)-, en un gel de agarosa LMP al 4\%. La muestra $018 \mathrm{D}$ representa el genotipo homocigoto negativo para la mutación 5382insC, con una banda a la altura de $214 \mathrm{pb}$, que se corresponde con el control negativo (MCF-7). Por otra parte, el control (+) D se muestra heterocigoto para la mutación 5382insC, con dos bandas de 234 pb y 214 pb para los alelos mutado y normal, respectivamente. Tanto la muestra como los controles ND, es decir sin enzima, se mostraron con un tamaño de 270 pb correspondiente al fragmento del exón 20 amplificado por PCR-mismatch.

Control (+) D: control positivo digerido; Control (+) ND: control positivo no digerido; MCF-7 D: control negativo digerido; MCF-7 ND: control negativo no digerido.

ADN directo: usado para probar la actividad de la enzima Ddel.

MPM 50 pb: marcador de peso molecular de $50 \mathrm{pb}$.

En la figura 3B se observan algunas muestras analizadas para la mutación 5382insC.

Con respecto a los receptores hormonales, se observó que en $46,7 \%$ de los tumores los receptores de estrógenos y progestágenos fueron negativos y que en $30 \%$ fueron positivos. Esto habría sido interesante verlo desde la perspectiva de la presencia de mutaciones en los genes BRCA1 o BRCA2, ya que estudios como el llevado a cabo por Palacios et al. lograron relacionar de forma significativa los tumores BRCA1 positivos con la negatividad de los receptores hormonales (receptores de estrógenos, $p=0,001$, y progestágenos, $p=0,002$ ) mediante análisis inmunohistoquímicos de tejidos de cáncer de mama (40); esto corresponde con las características fenotípicas de tumores $B R C A 1$ ya descritas como de tipo epitelial basal y de alto grado.

Aunque en este estudio en particular el grado histológico Bloom-Richardson más frecuente fue moderado $(46,7 \%)$, valdría la pena extender el análisis mutacional en esta cohorte de pacientes, no sólo a la totalidad del gen BRCA1 sino también al BRCA2, dadas las características inmunohistoquímicas ya referidas.

Teniendo en cuenta que las edades promedio de diagnóstico (49,6 años) y de la menopausia $(49,8$ años) coinciden en esta cohorte, es decir, que el diagnóstico se hizo alrededor de los 50 años o antes, y que la mayoría de cánceres de mama en general se desarrollan en la posmenopausia (el riesgo aumenta con la edad), se puede apoyar con nuestra muestra que cuando existe una historia familiar de cáncer de mama o de otros tipos, la edad de presentación es más temprana en los nuevos afectados; esto estaría a favor de una susceptibilidad genética de tipo hereditaria.

La prevalencia de mutaciones en los genes $B R C A$ depende de la población estudiada, los criterios de selección de las pacientes y los métodos de análisis moleculares empleados. Para la realización de este estudio, se empleó el método de PCR-mismatch, ya descrito en otras poblaciones como Chile, Alemania y ashkenazis 
(15,20,21,38); el método de PCR-mismatch en este estudio resultó ser sencillo de realizar y de analizar, más aun, fue reproducible y se pudo validar para el exón 2 mediante secuenciación directa. Otros métodos pueden usarse en el abordaje de los genes BRCA y su elección debe basarse en el tipo de mutación que se quiera buscar y en la sensibilidad que se quiera obtener en el proceso.

En los estudios realizados hasta la fecha no se han identificado otros genes de alta penetrancia diferentes a los $B R C A$; teniendo en cuenta que estos genes explican sólo una proporción de los cánceres de mama heredo-familiar, se propone que este cáncer es poligénico y que existirían muchos loci involucrados en su desarrollo, de los cuales, cada uno tendría un pequeño efecto en el riesgo para la enfermedad.

Easton et al. (41) identificaron cinco loci relacionados con el cáncer de mama en un estudio de asociación mediante polimorfismos de nucleótido único (single nucleotide polymorphisms, SNP), en el que se determinaron posibles genes causales, en su mayoría relacionados con mecanismos de control del crecimiento celular y señalización, en familias con dos o más casos de cáncer de mama en primer grado; este hallazgo genera nuevas expectativas con respecto a la investigación en genética del cáncer de mama, pues, a diferencia de este estudio, los anteriores se centraron en analizar genes de reparación y síntesis, y del metabolismo de hormonas sexuales.

Este estudio pretende motivar a la comunidad científica para continuar con la investigación de los aspectos moleculares del cáncer de mama, dirigida especialmente a la población de riesgo para la enfermedad, con el objeto de ofrecer un abordaje más integral y brindar mayores posibilidades de supervivencia y una mejor calidad de vida e, incluso, de curación.

Para el desarrollo de próximos estudios, se recomienda que se tengan en cuenta los criterios de inclusión de alto riesgo para ser portador de mutaciones en estos genes, como los manejados por U.S. Preventive Services Task Force (42) y por Stoppa-Lyonnet (43); la U.S. Preventive Services Task Force considera "al menos dos familiares en primer grado con cáncer de mama y/o cáncer de ovario" y, Stoppa-Lyonnet, "dos familiares en primer grado afectados: uno o más con cáncer de mama antes de los 41 años o con cáncer de ovario a cualquier edad; tres o más familiares en primer o segundo grado con cáncer de mama u ovario".

Asimismo, se sugiriere lo siguiente: i) ampliar el tamaño de la muestra con el objetivo de obtener resultados con mayor significancia estadística; ii) estudiar una mayor proporción del gen $B R C A 1$ e incluir el gen BRCA2, con el fin de lograr la caracterización de su perfil genético en la población de riesgo, y iii) contemplar, en los análisis moleculares en familias de cáncer de mama, el estudio no sólo de genes de alta penetrancia (BRCA, PTEN, ATM, P53), sino de nuevos genes identificados como asociados a esta patología en familias de alto riesgo.

Según nuestro conocimiento, éste es el primer estudio sobre cáncer de mama heredo/familiar en Bucaramanga, Santander, que pretende indagar sobre los aspectos genéticos relacionados.

\section{Agradecimientos}

Agradecemos a David Cregeen del NE Thames Regional Molecular Genetics Lab, London, UK, por su colaboración en cuanto a las técnicas de laboratorio y donación de controles positivos; a Adriana Castillo y al personal del Laboratorio de Genética de la Universidad Industrial de Santander, a la Junta Médica-Oncológica del Hospital Universitario de Santander y a todas las pacientes que participaron en el estudio y a sus familias.

\section{Conflicto de intereses}

Los autores declaramos que no existe conflicto de intereses.

\section{Financiación}

Maestría en Ciencias Básicas Biomédicas y Laboratorio de Genética de la Universidad Industrial de Santander

\section{Referencias}

1. Parkin DM. International variation Oncogene. 2004; 23:6329-40. 
2. Stewart SL, King JB, Thompson TD, Friedman C, Wingo PA. Cancer mortality surveillance--United States, 1990-2000. MMWR Surveill Summ. 2004;53:1-108.

3. Hormiga C, Rodríguez L, Uribe C. Análisis de la situación de las enfermedades neoplásicas en Santander. Revista del Observatorio de Salud Pública de Santander. 2006;2:4-30.

4. Miki Y, Swensen J, Shattuck-Eidens D, Futreal PA, Harshman K, Tavtigian S, et al. A strong candidate for the breast and ovarian cancer susceptibility gene BRCA1. Science. 1994;266:66-71.

5. Wooster R, Bignell G, Lancaster J, Swift S, Seal S, Mangion $\mathrm{J}$, et al. Identification of the breast cancer susceptibility gene BRCA2. Nature. 1995;378:789-92.

6. Easton D, Ford D, Peto J. Inherited susceptibility to breast cancer. Cancer Surv. 1993;18:95-113.

7. Marcus JN, Watson P, Page DL, Narod SA, Lenoir GM, Tonin $\mathbf{P}$, et al. Hereditary breast cancer: pathobiology, prognosis, and BRCA1 and BRCA2 gene linkage. Cancer. 1996;77:697-709.

8. Ford D, Easton DF, Stratton M, Narod S, Goldgar D, Devilee $\mathbf{P}$, et al. Genetic heterogeneity and penetrance analysis of the BRCA1 and BRCA2 genes in breast cancer families. The Breast Cancer Linkage Consortium. Am J Hum Genet. 1998;62:676-89.

9. National Human Genome Research Institute. The Breast Cancer Information Core Database, 2007. [Fecha de consulta: agosto de 2006 y marzo de 2007]. Disponible en: http://research.nhgri.nih.gov/bic/

10. Bar-Sade RB, Kruglikova A, Modan B, Gak E, HirshYechezkel G, Theodor L, et al. The 185delAG BRCA1 mutation originated before the dispersion of Jews in the diaspora and is not limited to Ashkenazim. Hum Mol Genet. 1998;7:801-5.

11. Diez O, Osorio A, Robledo M, Barroso A, Domenech $\mathbf{M}$, Cortés $\mathrm{J}$, et al. Prevalence of BRCA1 and BRCA2 Jewish mutations in Spanish breast cancer patients. $\mathrm{Br} \mathrm{J}$ Cancer. 1999;79:1302-3.

12. Neuhausen SL, Mazoyer S, Friedman L, Stratton M, Offit K, Caligo A, et al. Haplotype and phenotype analysis of six recurrent BRCA1 mutations in 61 families: results of an international study. Am J Hum Genet. 1996;58:271-80.

13. Roa BB, Boyd AA, Volcik K, Richards CS. Ashkenazi Jewish population frequencies for common mutations in BRCA1 and BRCA2. Nat Genet. 1996;14:185-7.

14. Struewing JP, Abeliovich D, Peretz T, Avishai N, Kaback MM, Collins FS, et al. The carrier frequency of the BRCA1 185delAG mutation is approximately 1 percent in Ashkenazi Jewish individuals. Nat Genet. 1995; 11:198-200.

15. Abeliovich D, Kaduri L, Lerer I, Weinberg N, Amir G, Sagi M, et al. The founder mutations 185delAG and 5382ins in BRCA1 and 6174delT in BRCA2 appear in $60 \%$ of ovarian cancer and $30 \%$ of early-onset breast cancer patients among Ashkenazi women. Am J Hum Genet. 1997;60:505-14.

16. Mullineaux LG, Castellano TM, Shaw J, Axell L, Wood ME, Diab S, et al. Identification of germline 185delAG BRCA1 mutations in non-Jewish Americans of Spanish ancestry from the San Luis Valley, Colorado. Cancer. 2003;98:597-602.

17. Schorge JO, Mahoney NM, Miller DS, Coleman RL, Muller CY, Euhus DM, etal. Germline BRCA1-2 mutations in non-Ashkenazi families with double primary breast and ovarian cancer. Gynecol Oncol. 2001;83:383-7.

18. Osorio A, Robledo M, Albertos J, Diez O, Alonso C, Baiget M, et al. Molecular analysis of the six most recurrent mutations in the BRCA1 gene in 87 Spanish breast/ ovarian cancer families. Cancer Lett. 1998;123:153-8.

19. Jara L, Ampuero S, Santibanez E, Seccia L, Rodríguez $\mathrm{J}$, Bustamante M, et al. BRCA1 and BRCA2 mutations in a South American population. Cancer Genet Cytogenet. 2006;166:36-45.

20. Jara L, Ampuero S, Seccia L, Bustamante M, Blanco R, Santibanez E, et al. Frequency of the 185delAG mutation in the BRCA1 gene in Chilean healthy women with family history of breast cancer. Rev Med Chil. 2002;130:1113-23.

21. Backe J, Hofferbert S, Skawran B, Dork T, Stuhrmann $\mathrm{M}$, Karstens $\mathrm{JH}$, et al. Frequency of BRCA1 mutation 5382insC in German breast cancer patients. Gynecol Oncol. 1999;72:402-6.

22. De Benedetti VM, Radice P, Pasini B, Stagi L, Pensotti V, Mondini P, et al. Characterization of ten novel and 13 recurring BRCA1 and BRCA2 germline mutations in Italian breast and/or ovarian carcinoma patients. Mutations in brief № 178. Hum Mutat. 1998;12:215.

23. Tommasi S, Crapolicchio A, Lacalamita R, Bruno $\mathrm{M}$, Monaco A, Petroni S, et al. BRCA1 mutations and polymorphisms in a hospital-based consecutive series of breast cancer patients from Apulia, Italy. Mutat Res. 2005;578:395-405.

24. van Der Looij M, Wysocka B, Brozek I, Jassem J, Limon J, Olah E. Founder BRCA1 mutations and two novel germline BRCA2 mutations in breast and/or ovarian cancer families from North-Eastern Poland. Hum Mutat. 2000;15:480-1.

25. Dufloth RM, Carvalho S, Heinrich JK, Shinzato JY, dos Santos CC, Zeferino LC, et al. Analysis of BRCA1 and BRCA2 mutations in Brazilian breast cancer patients with positive family history. Sao Paulo Med J. 2005;123:192-7.

26. Torres D, Rashid MU, Gil F, Umana A, Ramelli G, Robledo JF, et al. High proportion of BRCA1/2 founder mutations in Hispanic breast/ovarian cancer families from Colombia. Breast Cancer Res Treat. 2007; 103: 225-32. 
27. Brose MS, Rebbeck TR, Calzone KA, Stopfer JE, Nathanson KL, Weber BL. Cancer risk estimates for BRCA1 mutation carriers identified in a risk evaluation program. J Natl Cancer Inst. 2002;94:1365-72.

28. Ford D, Easton DF, Bishop DT, Narod SA, Goldgar DE. Risks of cancer in BRCA1-mutation carriers. Breast Cancer Linkage Consortium. Lancet. 1994;343:692-5.

29. Struewing JP, Hartge P, Wacholder S, Baker SM, Berlin M, McAdams M, et al. The risk of cancer associated with specific mutations of BRCA1 and BRCA2 among Ashkenazi Jews. N Engl J Med. 1997;336:1401-8.

30. Thompson D, Easton DF. Cancer Incidence in BRCA1 mutation carriers. J Natl Cancer Inst. 2002;94:1358-65.

31. Miller SA, Dykes DD, Polesky HF. A simple salting out procedure for extracting DNA from human nucleated cells. Nucleic Acids Res. 1988;16:1215.

32. Elstrodt F, Hollestelle A, Nagel JH, Gorin M, Wasielewski $\mathrm{M}$, van den Ouweland $\mathrm{A}$, et al. BRCA1 mutation analysis of 41 human breast cancer cell lines reveals three new deleterious mutants. Cancer Res. 2006;66:41-5.

33. Kutner SE. Breast cancer genetics and managed care. The Kaiser Permanente experience. Cancer. 1999;86:2570-4.

34. Ikeda N, Miyoshi Y, Yoneda K, Shiba E, Sekihara Y, Kinoshita M, et al. Frequency of BRCA1 and BRCA2 germline mutations in Japanese breast cancer families. Int J Cancer. 2001;91:83-8.

35. Peto J, Collins N, Barfoot R, Seal S, Warren W, Rahman N, et al. Prevalence of BRCA1 and BRCA2 gene mutations in patients with early-onset breast cancer. J Natl Cancer Inst. 1999;91:943-9.
36. Ruiz-Flores $\mathbf{P}$, Calderón A, Barrera-Saldaña H. Breast Cancer Genetics BRCA1 and BRCA2: Main susceptibility genes. Revista de Investigación Clínica. 2001;53:46-64.

37. Trincado P, Fardella C, Mayerson D, Montero L, O'Brien A, Barrueto K, et al. Prevalence of the $185 \mathrm{Ag}$ deletion of the BRCA1 gene in Chilean women with breast neoplasm. Rev Med Chil. 1999;127:19-22.

38. Jara L, Ampuero S, Seccia L, Bustamante M, Blanco R, Ojeda JM. Analysis of 5382insC (BRCA1) and 6174delT (BRCA2) mutations in 382 healthy Chilean women with a family history of breast cancer. Biol Res. 2002;35:85-93.

39. Mantilla A, Vesga B, Insuasty J. Registro de Cáncer, Unidad de Oncología, Hospital Universitario Ramón González Valencia, Bucaramanga, Colombia. (19961999). MedUNAB. 2006;9:14-9.

40. Palacios J, Honrado E, Osorio A, Cazorla A, Sarrio D, Barroso A, et al. Immunohistochemical characteristics defined by tissue microarray of hereditary breast cancer not attributable to BRCA1 or BRCA2 mutations: differences from breast carcinomas arising in BRCA1 and BRCA2 mutation carriers. Clin Cancer Res. 2003;9:3606-14.

41. Easton DF, Pooley KA, Dunning AM, Pharoah PD, Thompson D, Ballinger DG, et al. Genomewide association study identifies novel breast cancer susceptibility loci. Nature. 2007;447:1087-93.

42. U.S. Preventive Services Task Force (USPSTF). Genetic risk assessment and BRCA mutation testing for breast and ovarian cancer susceptibility: recommendation statement. Ann Intern Med. 2005;143:355-61.

43. Stoppa-Lyonnet D, Laurent-Puig P, Essioux L, Pages $\mathrm{S}$, Ithier G, Ligot $\mathrm{L}$, et al. BRCA1 sequence variations in 160 individuals referred to a breast/ovarian family cancer clinic. Institut Curie Breast Cancer Group. Am J Hum Genet. 1997;60:1021-30. 